\section{Nidoviruses in snakes in Europe}

\section{Rachel E. Marschang, Ekaterina Kolesnik, Elisabeth Müller}

Laboklin GmbH \& Co. KG, Bad Kissingen, Germany

\section{OBJECTIVES}

Nidoviruses are single stranded RNA viruses that were first described in reptiles in 2014 . They can cause severe respiratory disease in numerous python species. The aim of this study was to determine the prevalence of these pathogens in samples from snakes submitted to a diagnostic laboratory in Europe.

\section{METHODS}

Samples from 1171 snakes were tested for the presence of nidoviruses between January 2016 and September 2018. The majority of samples were oral swabs. Each sample was tested for the presence of nidoviral RNA by PCR.
Samples were from a wide range of species, including ball pythons (Python regius, 343 animals) and green tree pythons (Morelia viridis, 214 animals). In many cases, the species was not specified.

\section{RESULTS}

A total of 300 of the snakes examined $(25.6 \%)$ were positive for nidoviruses, including $76(22.2 \%)$ ball pythons and $69(32.2 \%)$ green tree pythons. There was a significant difference in the likelihood of virus detection between these two species ( $p=0.008329$ ). Evaluation of the results over time showed no seasonal pattern in the detection rates. Positive samples were obtained from animals in Great Britain, Germany, Austria, Switzerland, Spain, France, Belgium, the Netherlands, Denmark, Poland, Sweden, Italy, and the Czech Republic.

\section{STATEMENT (CONCLUSIONS)}

This study shows that nidoviruses are important pathogens in pythons in Europe and are widespread throughout the continent. Interestingly, green tree pythons appear to be more commonly infected than other python species.

\section{Patterns of antimicrobial prescription in rabbits attending first-opinion practices in the UK}

\section{Samuel Pearce, Dr Fernando Sánchez-Vizcaíno}

Bristol Veterinary School, University of Bristol, Bristol, United Kingdom

\section{OBJECTIVES}

To describe antimicrobial prescription patterns in rabbits attending first-opinion practices in the UK.

\section{METHODS}

Electronic health records (EHRs) were collected through the Small Animal Veterinary Surveillance Network from March 2014 to June 2018. EHRs included information on the consultation date, animal signalment and main presenting complaint. EHRs further included product descriptions as defined by individual practices and, where possible, further product information. Product descriptions with no pharmaceutical information recorded were reviewed and if an antimicrobial was identified, it was manually defined with reference to the Veterinary Medicines Directorate's Product Information Database for veterinary authorised products, and the electronic Medicines Compendium for human authorised products. Descriptive statistics and data visualisation were conducted using $\mathrm{R}$ language.

\section{RESULTS}

A total of 74,621 rabbit consultations were gathered from 498 veterinary premises nationwide. During the study, $21.7 \%$ of rabbits were exposed to at least one antimicrobial. A negative trend was observed for the prescription rate over the 4-year period. Antimicrobials were most commonly prescribed in respiratory disease-related consultations ( $67.7 \%$ of respiratory consultations). Systemic and topical antimicrobial agents represented $63.7 \%$ and $35.9 \%$ of all prescriptions, respectively, the rest being unidentified. The most commonly used antimicrobials were fluoroquinolones ( $49.8 \%$ of all prescriptions). Finally, $12.7 \%$ of all prescriptions were human authorised.

\section{STATEMENT (CONCLUSIONS)}

The study suggests a reduction in antimicrobial prescribing in rabbits, which may reflect the success of awareness campaigns. This study will form the basis of future research into antimicrobial prescribing and antimicrobial resistance in rabbits, providing valuable data that the profession needs to ensure antimicrobials are used responsibly. 